(C) Facultad de Ciencias Biológicas UNMSM

\title{
Diversidad, abundancia y conservación de aves en un agroecosis- tema del desierto de Ica, Perú
}

\section{Diversity, abundance and conservation of birds in an agroecosystem in the Ica desert, Peru}

Letty Salinas ${ }^{1}$, César Arana ${ }^{1}$ y Víctor Pulido²

\begin{abstract}
1 Universidad Nacional Mayor de San Marcos, Museo de Historia Natural y Facultad de Ciencias Biológicas. Apartado 140434, Lima 14, Perú.

E-mail: Letty Salinas Isalinass@unmsm.edu.pe

2 Escuela de Postgrado de la Universidad Inca Garcilaso de la Vega
\end{abstract}

\section{Resumen}

El presente trabajo investiga el impacto de la actividad agrícola en la costa peruana sobre la biodiversidad de aves. El estudio comprende el monitoreo de aves de dos terrenos de cultivo del valle de Ica, dedicados al cultivo de espárragos y uvas, desde enero-2004 hasta enero-2006. Evaluamos ocho tipos de hábitats distribuidos en un total de 1288 ha. Durante todo el periodo de estudio registramos 93 especies de aves. La abundancia, riqueza y diversidad fue mayor en los veranos. La mayor abundancia ocurrió en los hábitats de esparragales, monte ribereño y cerco. La mayor riqueza y diversidad ocurrieron en enero-2006 en el alfalfar con huarangos y cercos vivos. Las especies más abundantes fueron las residentes Zenaida meloda (6,6 ind./ha), Pygochelidon cyanoleuca $(5,9)$ y la migratoria Hirundo rustica $(5,9)$. Registramos 12 especies migratorias. Desde el punto de vista conservacionista, el número de especies catalogadas en algún grado de amenaza, a nivel nacional e internacional, es muy alto. Podemos destacar el vulnerable Xenospingus concolor, el endémico nacional Colaptes atricollis y las especies de desierto Geositta peruviana, Sporophila simplex y Burhinus superciliaris. Este estudio demuestra la importancia de agroecosistemas en la conservación de la biodiversidad del Desierto Costero Peruano, particularmente si la empresa realiza una política de respecto al medioambiente

Palabras claves: Agroecosistemas, aves, Desierto Costero Peruano, conservación, Ica, Perú.

\section{Abstract}

Present work investigates the impact of the agricultural activity in the Peruvian coast on the biodiversity of birds. The study includes the monitoring of birds in asparagus and grapes farms of Ica valley, from January-2004 to January-2006. We evaluated eight types of habitats distributed in a total of 1288 has. Throughout the period of study we registered 93 species of birds. The abundan$\mathrm{ce}$, richness and diversity were greater in the summers. The greater abundance happened in the habitats of land of asparagus, river brushwood and live fences. The greater richness and diversity happened in January-2006 in alfalfa cultures with huarangos and live fences. The most abundant species were the residents, as Zenaida meloda (6,6 ind./it has), Pygochelidon cyanoleuca $(5,9)$ and the migratory Hirundo rustica $(5,9)$. We registered 12 migratory species. From the conservationist point of view, the number of species catalogued in some degree of threat, at national and international level, is very high. We can emphasize the vulnerable Xenospingus concolor, the Peruvian endemic Colaptes atricollis and the species of desert Geositta peruviana, Sporophila simplex and Burhinus superciliaris. This study demonstrates the importance of agroecosystems in the conservation of the Peruvian Coastal Desert biodiversity, in particular if the enterprise carries out a policy of respect to environment.

Keywords: Agroecosystems, birds, Peruvian Coastal Desert, Conservation, Ica, Peru.

\section{Introducción}

El crecimiento de la agricultura en las pasadas décadas ha sido impresionante en todo el planeta, produciendo un intenso impacto sobre los ecosistemas terrestres y acuáticos (Tilman, 1999; Tilman et al., 2001) y causando que la superficie de la Tierra sea dominada por agroecosistemas (Ormerod \& Watkinson, 2000; Defries et al., 2004).

Tilman (1999) señala que los impactos mas importantes sobre la biodiversidad se deben al deliberado mantenimiento de ecosistemas altamente simplificados, disturbados y enriquecidos con nutrientes, como suelen ser las mayores extensiones de cultivos en la actualidad. Es así que en la actualidad cuatro plantas, cebada, maíz, arroz y trigo, naturalmente «raras» sean las dominantes en el planeta.

Este hecho hace necesario el establecimiento de programas de investigación multidisciplinarios que desarrollen métodos agrícolas que puedan alimentar a la creciente población humana pero que a la vez preserven los servicios vitales para la humanidad que proveen los ecosistemas silvestres (Myers, 1996).

Si bien la pérdida de hábitats silvestres es la principal causa de la pérdida de la biodiversidad mundial (Heywood \& Watson, 1995; Myers et al., 2000), se ha señalado que algunos agroecosistemas pueden conservar una sustancial parte de la biodiversidad de su anterior ecosistema natural y servir como zonas buffer y complementarias de las áreas protegidas (Vandermeer \& Perfecto, 1997; Curran et al., 2004; Schroth et al., 2004; Belfrage et al., 2005).

Estudios recientes han examinado la influencia de los agroecosistemas sobre una variedad de taxones incluyendo plantas (Belfrage et al., 2005; Fujisaka et al., 1998; Harvey et al., 2005; Mayfield \& Daily, 2005), escarabajos (Estrada et al., 1999, 1998; Settele et al., 1993), mariposas (Horner-Devine et al., 2003), polillas (Ricketts et al., 2001; Settele et al., 1993), aves 
(Belfrage et al., 2005; Daily et al., 2001) y monos (Estrada et al., 1999). La mayoría de estos estudios han sido desarrollados en bosques tropicales de diferentes lugares del mundo.

Desde un punto de vista agronómico la relación entre los cultivos y las aves es controversial, como ha sido reconocido desde hace más de un siglo (Judd, 1898). Por un lado, la abundancia de ciertos tipos de alimentos, sitios para reproducción, protección y eliminación de depredadores dentro de los agroecosistemas, ocasiona que algunas especies de aves aumenten sus poblaciones y se conviertan en plagas que diezman los cultivos (Poleo \& Fuentes, 2005; Valencia, 1985; Cuevas, 2001; Reyes, 1985; Robles et al., 2003). Sin embargo, otras aves sirven como eficientes controladores de insectos dañinos para los cultivos, prestan servicio de polinización y disminuyen significativamente el éxito reproductivo de malezas consumiendo sus semillas (Myers, 1996; Robles et al., 2003; Cuevas, 2001). Adicionalmente, las aves pueden servir como eficaces indicadoras de la calidad del ambiente en los agroecosistemas (Belgrafe et al., 2005).

En el Perú muy recientemente se están ejecutando estudios de aves en relación a campos de cultivo. En el departamento de Arequipa (sur del país) en 1999 se evalúo el daño que las aves de la Irrigación Majes infringían a los cultivos de vid (Málaga \& Rayan, 2003) y en el 2003 el daño que las aves acuáticas del Santuario Nacional Lagunas de Mejía ocasionaban a los cultivos de arroz adyacentes (Pilares \& Arenas, 2004). En la parte altoandina del Perú central se ha evaluado a las aves como plagas de cultivos de quinua (Robles et al., 2003). Estas investigaciones fueron conducidas en áreas agrícolas pequeñas, no mayores de 18 ha, en periodos de evaluación cortos e irregulares, dando como resultado el registro de tan sólo una a tres decenas de especies de aves.

En los últimos años una creciente agroindustria de exportación ha incrementado el área cultivada en el desierto costero del Perú. Este desierto tiene particularidades tanto biológicas (alto endemismo y baja protección) como sociales (principales poblaciones humanas y alto desarrollo urbano e industrial). Ica es uno de los departamentos con mayor desarrollo agroindustrial, por lo que es necesario el establecimiento de estudios de largo término acerca del impacto de esta actividad económica sobre la biodiversidad. Nuestra investigación busca documentar la influencia de un agroecosistema administrado con responsabilidad ambiental sobre la diversidad de la avifauna silvestre. Con esta finalidad se llevo a cabo un monitoreo de la avifauna desde enero de 2004 hasta enero de 2006 en dos campos de cultivo del valle de Ica, dedicados al cultivo de espárragos y uvas, en una extensión total de más de mil hectáreas.

\section{Métodos \\ Área de estudio}

Las áreas evaluadas son dos campos agrícolas de una de las mayores empresas exportadoras de espárragos del Perú, la Sociedad Agrícola Drokasa (AGROKASA). Estos dos fundos, Santa Rita y La Catalina, se encuentran en la costa del departamento de Ica, al sur de Lima, dentro del dominio morfoclimático o ecorregión del desierto del Pacífico (14 $07^{\circ}-14^{\circ} 11^{\prime} S$ y $75^{\circ} 38^{\prime}$ $75^{\circ} 43^{\prime} \mathrm{W}$ ) (Fig. 1). En su mayor parte esta región está conformada por planicies y colinas bajas que se elevan en dirección Este. Esta región se caracteriza por tener un clima desértico cálido con temperaturas elevadas durante el día $\left(23{ }^{\circ} \mathrm{C}\right.$ de temperatura media anual), el que se acentúa por la escasa nubosidad, a pesar de eso la humedad relativa generalmente está por encima de $60 \%$, pero las precipitaciones son muy poco frecuentes. Los vientos son de moderados a fuertes, pero se tornan más intensos cuando aparecen los vientos conocidos como Paracas, de origen oceánico.

El fundo Santa Rita se encuentra a la altura del kilómetro 312,4 de la carretera Panamericana Sur, cerca de los distritos de Tate y Pueblo Nuevo, mientras que el Fundo La Catalina se encuentra en el desvío del kilómetro 316, hacia el Este en la Pampa de los Castillos en los distritos de Pueblo Nuevo y Pachacutec (Fig. 1).

El Fundo Santa Rita abarca una extensión de 188 ha y el Fundo La Catalina de 1100 ha. En ambos fundos se desarrollan actividades fundamentalmente agrícolas utilizando riego por goteo lo que permite la conservación de amplias extensiones de suelo desértico. La vegetación silvestre típica del área alrededor de los fundos estaba representada por un desierto con árboles y arbustos xerofíticos muy dispersos, principalmente de Prosopis pallida «huarango», Parkinsonia aculeata «azote de cristo», Cercidium praecox «palo verde», Bulnesia retama «calato» y la endémica de Ica y Lima, Tecoma guarume «cahuato»; también un monte ribereño con abundancia de Arundo donax «caña brava», Tessaria integrifo-

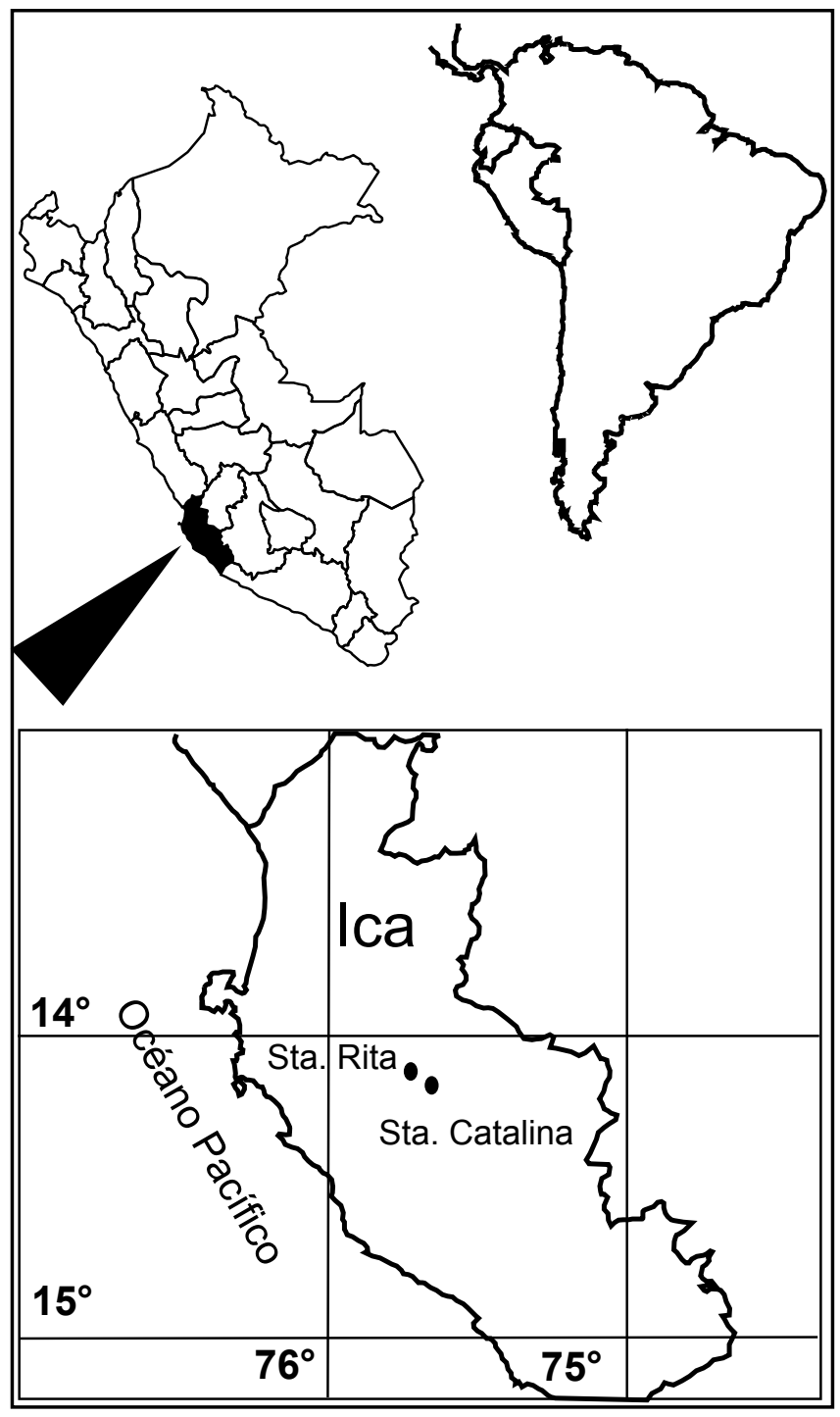

Figura1. Mapa de ubicación de los fundos evaluados. 
lia «pájaro bobo» y Baccharis salicifolia «chilco», así como Acacia macracantha «espino», finalmente un pequeño humedal costero formado por efecto de la irrigación agrícola.

Los tipos de hábitats para aves observados en los fundos fueron:

1. Viñedos: Incluyó las extensiones de terrenos de cultivo de «vid» Vitis vinifera, caracterizados por poseer estructura arbustiva debido a la forma en que se cultivan, haciendo crecer a la planta sobre soportes de aproximadamente dos metros, quedando reducido el paso de luz hacia el suelo, el cual frecuentemente es liberado de malezas.

2. Esparragales: Comprendió extensiones de terrenos de cultivos de «espárrago» Asparagus officinalis. Este hábitat presentó diferentes estados de desarrollo. En periodo de cosecha sólo se encuentran los brotes de la planta, mientras que durante el periodo de descanso, las plantas son de porte alto (hasta 1,50 $\mathrm{m}$ aproximadamente), con follaje desarrollado, flores y frutos. Este hábitat es exclusivamente de hierbas e incluye en ciertos periodos mayor diversidad de malezas entre los espárragos.

3. Cercos vivos: estuvieron conformados por cercos principalmente de plantas leñosas y espinosas llamadas comúnmente «aromo» Acacia cf. horrida y otros arbustos los que se alternan en algunos casos con árboles frutales como mango, pacae, plátano, etc. También se encuentran algunos árboles nativos de Prosopis pallida llamada localmente «huarango» y «algarrobo» en otras regiones del Perú. La altura del cerco es variable aproximadamente de dos metros en promedio, alcanzando en algunos casos seis metros o más en sectores pequeños.

4. Monte ribereño: Comprendió la vegetación que rodea los canales San Agustín y Tacaraca que atraviesan Santa Rita, estos canales son de importante volumen y presentaban características similares a los brazos naturales del río Ica y la zona colindante con éste. Este hábitat presentó una vegetación compleja con abundante presencia de Arundo donax «caña brava» y Salix humboldtiana «sauce». Los canales en algunos periodos del año son liberados de la vegetación herbácea.

5. Jardines e Instalaciones: Comprendió el área administrativa de ambos fundos, rodeadas de jardines y de algunos árboles y arbustos, cuya composición florística fue predominantemente ornamental o de plantas frutales.

6. Desierto: Zona desértica de La Catalina estaba caracterizada por la presencia de vegetación silvestre, dominada por árboles pequeños y dispersos de Parkinsonia aculeata «azote de cristo», Cercidium praecox «palo verde» y Acacia macracantha «espino». También se encuentran arbustos de Bulnesia retama «calato» y Tecoma guarume «cahuato». Parte del área correspondió a un abanico aluvial donde se concentra la vegetación xerofítica que utiliza el agua del subsuelo. En la zona desértica AGROKASA ha establecido un área intangible de conservación de vida silvestre.

7. Zona de Eucalipto: estaba ubicada en el Fundo Santa Rita, formada por un pequeño grupo de árboles de eucaliptos, desprovistos de vegetación herbácea conservando el suelo totalmente arenoso y disponible para las aves típicas del ambiente desértico.

8. Zona de alfalfar con huarangos: estaba ubicada en el fundo La Catalina, y presentaba por lo menos dos agregaciones importantes de «huarangos» Prosopis pallida adultos rodeadas de cultivos de alfalfa y hortalizas. Esta zona constituía un área de concentración de aves debido a su importante cobertura arbórea.

\section{Métodos de censo y análisis de datos cuantitati- vos}

Desde enero de 2004 a enero de 2006 se evalúo cuantitativamente la avifauna, en cada tipo de hábitat presente en los fundos. Un primer reconocimiento de la avifauna fue realizado en abril de 2003. Tres tipos de hábitats no fueron accesibles desde el inicio del estudio, y fueron evaluados, en el caso del Desierto y del alfalfar con Huarangos desde agosto de 2004 y la zona de eucaliptos sólo en enero de 2006.

Para el inventario se registraron las aves presentes en los fundos mediante la observación directa con binoculares y una búsqueda activa visual y auditiva. Para el censo se empleó el método de conteo de puntos (Reynolds et al., 1980; Buckland, 1987; Bibby et al., 1985; Bibby \& Charlton, 1991). Este método de censo es uno de los más eficientes para calcular la abundancia, en especial cuando la evaluación comprende una amplia área de estudio, diferentes tipos de hábitat (Bibby et al., 1993) y las aves difieren en muchas características como organización social, tamaño y hábitos (Koskimies \& Väisänen, 1991). Asimismo, este método permite completar el inventario y evaluar los aspectos generales de los hábitats en cada punto de censo y registrar a las especies tanto por la observación directa como por sus huellas, nidos, cantos y otras señales.

Los puntos de censo fueron ubicados en mapas de los fundos siguiendo un diseño estratificado al azar donde, cada estrato estaba representado por un tipo de hábitat y la distancia mínima entre ellos fue de $100 \mathrm{~m}$. En el campo se establecieron rutas de acceso a los puntos de censo siguiendo caminos adyacentes o lugares libres de modo que el muestreo a largo plazo pudiera ser viable, sin que éste afecte o se vea afectado por el desarrollo de la actividad agrícola.

En cada tipo de hábitat se ubicó alrededor de diez puntos de censo, los que fueron georeferenciados y caracterizados para su seguimiento durante todo el periodo de estudio. En cada punto de censo se dejó transcurrir un corto tiempo (no menor de 1 minuto) luego de la llegada del censador al lugar a ser evaluado, para que cese el disturbio que originó y detectar a las aves presentes durante un tiempo determinado (tiempo de conteo). Respecto al tiempo de conteo en la literatura existe un rango preferente que va desde 2 a 20 minutos (Bibby et al., 1993). Se seleccionó el periodo de 10 minutos como el más conveniente, por cuanto este periodo de tiempo es lo suficientemente corto para no confundirse con el desplazamiento de las aves gregarias ni con las que se desplazan muy activamente, y lo suficientemente largo como para registrar a aves solitarias, poco conspicuas o de vocalización discontinua. Este periodo de conteo ha sido establecido como conveniente en otros ambientes desérticos de la costa peruana (Salinas \& Arana, comunicación personal).

Entre las diferentes variantes de este método, se seleccionó el método de Conteo de Puntos No Limitado a la Distancia (Bibby et al., 1993). En este método no se establece un radio fijo al inicio del trabajo de campo ya que se registra a todas las aves que se pueden detectar en el área, pues el censador hace las veces de un centro de detección de actividad dentro de un área semiesférica.

La determinación de un valor de radio de avistamiento (r) se realizó a partir de los datos de distancias obtenidos en el muestreo (Bibby et al., 1993). Para los fundos se determinó valores de r 


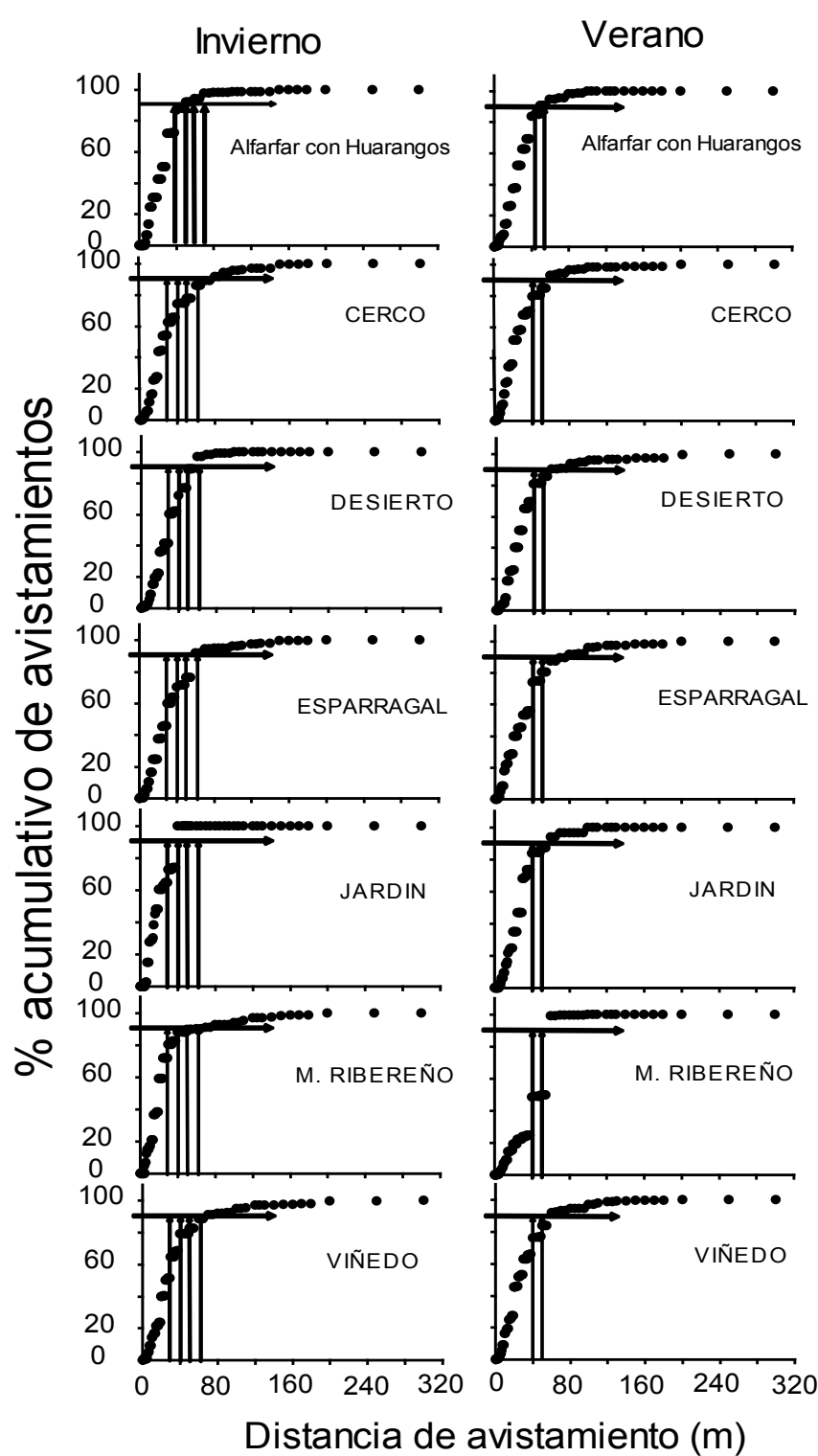

Figura 2. Determinación de los valores del radio de avistamiento (señalados con flechas rojas) para los diferentes hábitats de los fundos, en los meses de invierno y verano.

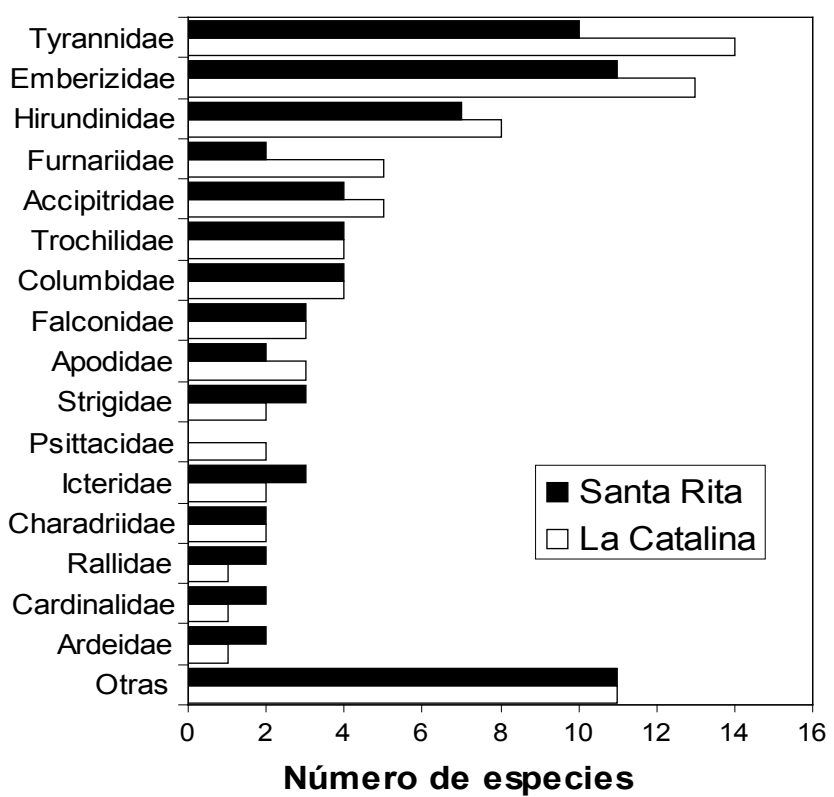

Figura 3. Número de especies por familias en cada uno de los fundos.
Tabla 1. Valores del radio de avistamiento $(r)$ en metros, para cada tipo de hábitat y época de evaluación.

\begin{tabular}{lcc}
\hline Tipo de Hábitat & \multicolumn{2}{c}{ Valores de $\mathbf{r}(\mathbf{m})$} \\
Invierno & Verano \\
\hline Alfalfar con huarangos & 50 & 50 \\
Cercos vivos & 70 & 60 \\
Desierto & 60 & 60 \\
Esparragales & 60 & 60 \\
Eucalipto & 40 & 60 \\
Jardines e Instalaciones & 60 & 60 \\
Monte ribereño & 70 & 60 \\
Viñedos &
\end{tabular}

utilizando un acercamiento gráfico del porcentaje acumulativo de avistamientos, determinando el radio como la distancia en la cual disminuye drásticamente este valor (Fig. 2). Para estas gráficas se utilizaron todos los avistamientos, discriminando los de los meses de invierno y verano (Fig. 2).

Los valores de $\mathrm{r}$ determinados para cada tipo de hábitat presente en los fundos y para cada época (invierno y verano) son resumidos en la Tabla 1. Este análisis se ha realizado considerando un total de 14256 individuos avistados, 2660 en los meses de invierno y 11596 en los de verano.

Los datos obtenidos fueron utilizados para caracterizar la avifauna de cada hábitat en: (a) composición taxonómica; (b) abundancia, utilizando la densidad por ha (Bibby et al., 1993) y (c) diversidad de especies, utilizando el índice de diversidad H' de Shannon-Wiener y las curvas de rarefacción (Magurran, 1991).

Adicionalmente, para completar el inventario y obtener registros fotográficos de las aves, un promedio de 25 redes fueron colocadas en cada fundo en los diferentes tipos de hábitats presentes.

\section{Resultados \\ Composición taxonómica}

La avifauna de los fundos Santa Rita y La Catalina en conjunto esta compuesta por 93 especies de aves incluidas en 29 familias y 12 órdenes (Tabla 2). En Santa Rita se encontraron 72 especies, mientras que en La Catalina 81 (Tabla 2). El Orden mejor representado es el de los Passeriformes con 13 Familias y 55 especies que constituye el $59,1 \%$ de las especies de avifauna observadas en este estudio. Las familias con mayores números de especies son Tyrannidae con 16 especies, Emberizidae con 14 especies e Hirundinidae con 8 especies (Fig. 3).

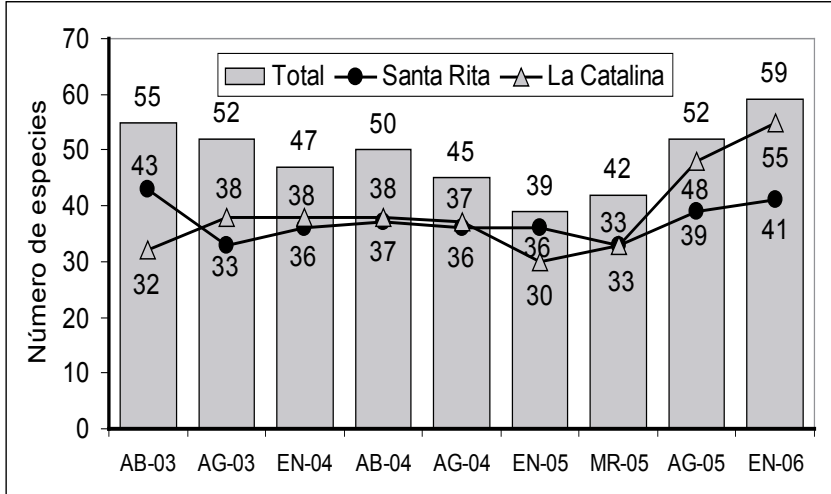

Figura 4. Número de especies por mes de evaluación, totales y valores de cada fundo por separado. 


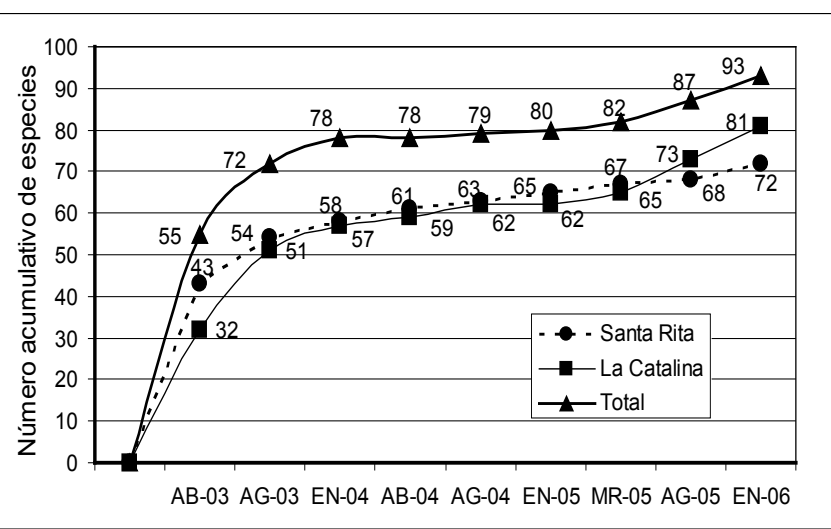

Figura 5. Número acumulativo de especies por periodo de evaluación en ambos fundos y en total.

La fluctuación del número de especies por fecha de evaluación presenta los valores más altos en abril y agosto de 2003, agosto de 2005 y el mayor valor (59 especies) en enero de 2006 (Fig. 4). La variación en la riqueza de especies en los dos fundos es similar. Sin embargo el incremento del número de especies, en agosto de 2005 y enero de 2006, es mayor en La Catalina, esto puede ser explicado por la evaluación de hábitats antes no incluidos (desierto y alfalfar con huarangos). La curva de acumulación de especies indica que no se esperaría un incremento significativo en futuras evaluaciones (Fig. 5).

Si bien, las especies de aves encontradas en los fundos, en su mayoría son típicas de los campos agrícolas de ambientes costeros de este departamento; se ha encontrado también que la composición de especies está fuertemente influenciada por aves que frecuentan la vegetación ribereña y los hábitats arbustivos y desérticos silvestres de alrededores de los fundos.

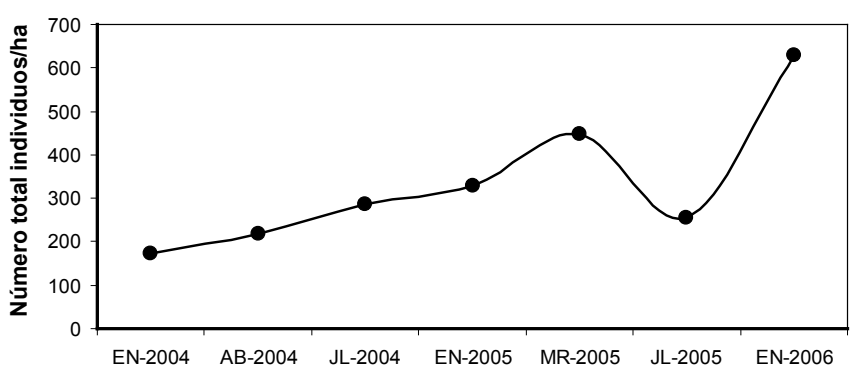

Figura 6. Número total de individuos por hectárea para cada periodo de evaluación

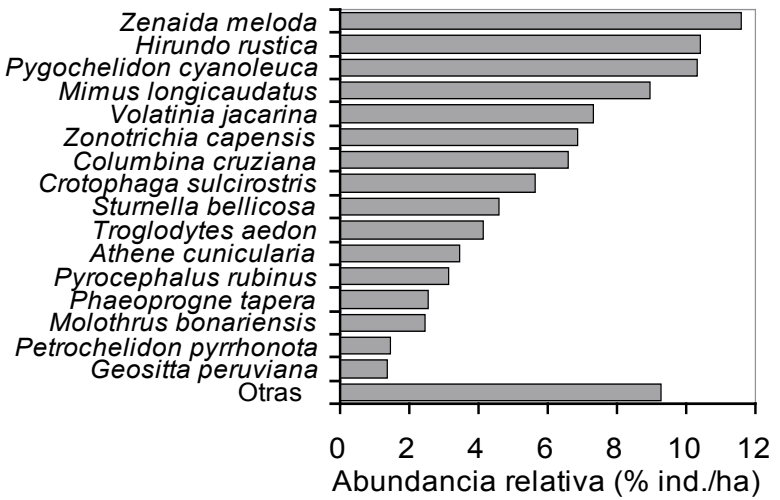

Figura 8. Abundancia relativa de las especies más abundantes en los fundos de AGROKASA en Ica.

\section{Abundancia}

La fluctuación de la abundancia muestra una tendencia al incremento en el tiempo, con picos de mayores valores coincidentes con los meses de verano (Enero de 2006 y, Enero y Marzo de 2005), fundamentalmente por la llegada de especies migratorias en ese periodo (Fig. 6). Por otro lado, se observa una fuerte disminución en julio de 2005 coincidiendo con una de las épocas invernales más frías.

La mayor abundancia ocurrió en esparragales (201 individuos/ ha, marzo de 2005), seguido por monte ribereño (185 ind./ha, enero de 2006) y cercos vivos (173 ind./ha, enero-2004; 172 ind./ha, enero de 2005). En todos los casos los valores mayores coincidieron con el verano (Fig. 7). Los viñedos presentaron los menores valores de abundancia en casi todos los periodos de evaluación.

Los mayores valores de abundancia de aves tienen diferentes causas en cada tipo de hábitat. En los esparragales la mayor abundancia esta relacionada con la mayor extensión que presentan en los fundos y con la frecuencia con que numerosas aves buscan alimento o refugio en ellos. Los cercos vivos y monte ribereño, con mayor complejidad estructural ofrecen a las aves lugares para buscar alimento posarse y anidar. Los viñedos presentan las menores abundancias debido a que en la mayor parte del año no ofrecen recursos atractivos para las aves y durante el periodo de fructificación se efectúan acciones de control de las aves con ahuyentadores sónicos y cetrería.

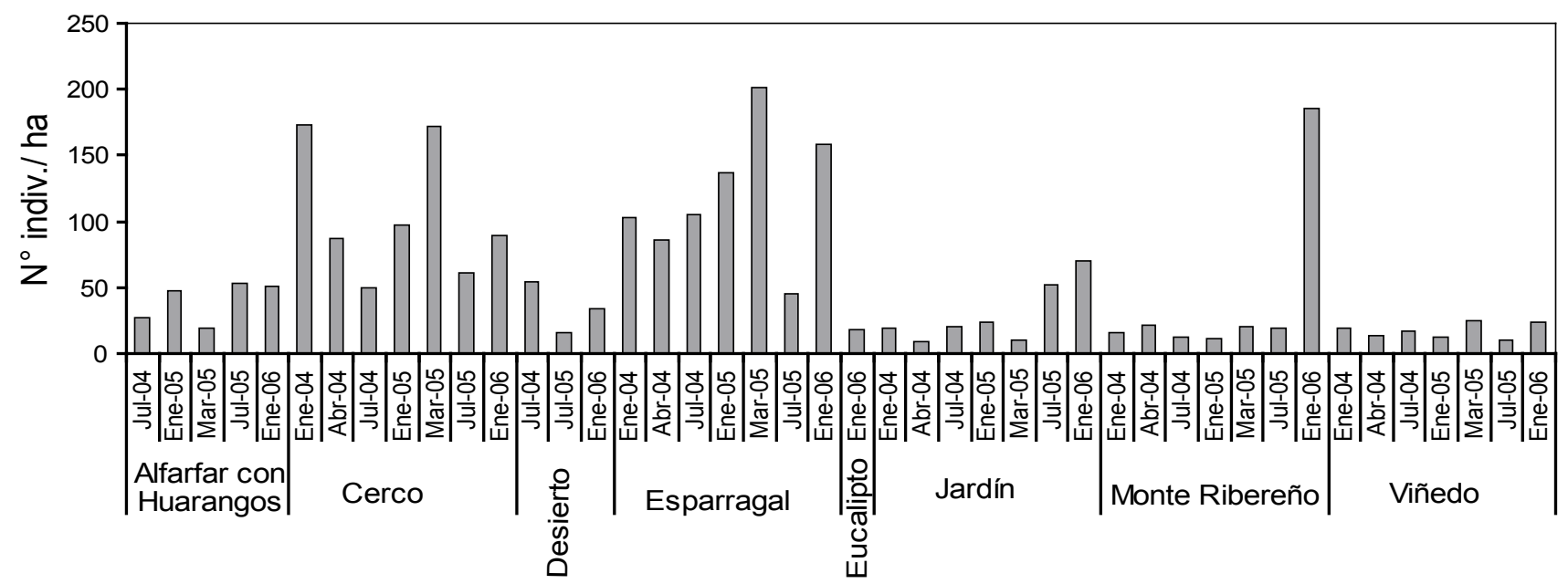

Figura 7. Número total de individuos por hectárea para cada periodo de evaluación y en cada tipo de hábitat evaluado. 
Tabla 2. Lista de especies de aves presentes en los fundos Santa Rita y La Catalina1.

\begin{tabular}{|c|c|c|c|c|c|}
\hline $\mathbf{N}^{\circ}$ & Nombre Científico & Nombre español & Nombre inglés & $\begin{array}{l}\text { Sta. } \\
\text { Rita }\end{array}$ & $\begin{array}{c}\mathrm{La} \\
\text { Catalina }\end{array}$ \\
\hline & Ardeidae & Garzas & Herons & & \\
\hline 1 & Ardea alba Linnaeus & Garza blanca grande & Great Egret & $\mathrm{x}$ & $\mathrm{X}$ \\
\hline \multirow[t]{2}{*}{2} & Egretta thula Molina & Garza blanca chica & Snowy Egret & $x$ & \\
\hline & Cathartidae & Gallinazos & New world vultures & & \\
\hline \multirow[t]{2}{*}{3} & Cathartes aura Linnaeus & Gallinazo cabeza roja & Turkey Vulture & $\mathrm{X}$ & $\mathrm{X}$ \\
\hline & $\begin{array}{l}\text { Accipitridae } \\
\end{array}$ & Gavilanes y aguilas & Hawks & & \\
\hline 4 & Circus cinereus Vieillot & Gavilán cenizo & Cinereous Harrier & $x$ & $\mathrm{X}$ \\
\hline 5 & Parabuteo unicinctus Temminck & Gavilán oscuro acanelado & Harris' (Bay-winged) Hawk & $x$ & $\mathrm{X}$ \\
\hline 6 & Geranoaetus melanoleucus Vieillot & Aguilucho pechinegro & Black-chested Buzzard-Eagle & & $\mathrm{X}$ \\
\hline 7 & Buteo platypterus Vieillot & Aguilucho ala ancha & Broad-winged Hawk & $\mathrm{x}$ & $x$ \\
\hline \multirow[t]{2}{*}{8} & Buteo polyosoma Quoy \& Gaimard & Aguilucho común & Variable (Red- backed) Hawk & $x$ & $X$ \\
\hline & Falconidae & Halcones & Falcons & & \\
\hline 9 & Falco sparverius Linnaeus & Cernícalo americano & American Kestrel & $\mathrm{X}$ & $\mathrm{X}$ \\
\hline 10 & Falco femoralis Temminck & Halcón aplomado & Aplomado Falcon & $\mathrm{X}$ & $x$ \\
\hline \multirow{2}{*}{11} & Falco peregrinus Tunstall & Halcón peregrino & Peregrine Falcon & $\mathrm{X}$ & $\mathrm{X}$ \\
\hline & Rallidae & Rascones y gallaretas & Rails & & \\
\hline 12 & Pardirallus sanguinolentus Swainson & Gallineta común & Plumbeous Rail & $\mathrm{X}$ & $\mathrm{X}$ \\
\hline \multirow{2}{*}{13} & Gallinula chloropus Linnaeus & Polla de agua & Common Moorhen & $\mathrm{X}$ & \\
\hline & Burhinidae & Alcaravanes & Thick-knees & & \\
\hline \multirow{2}{*}{14} & Burhinus superciliaris Tschudi & Huerequeque & Peruvian Thick-knee & $\mathrm{X}$ & $X$ \\
\hline & Charadriidae & Chorlos & Plovers & & \\
\hline 15 & Charadrius vociferus Linnaeus & Chorlo gritón & Killdeer & $\mathrm{X}$ & $\mathrm{X}$ \\
\hline 16 & Charadrius alexandrinus Linnaeus & Chorlo nevado & Snowy (Kentish) Plover & $\mathrm{X}$ & \\
\hline \multirow{2}{*}{17} & Oreopholus ruficollis Wagler & Chorlo de campo & Tawny-throated Dotterel & & $X$ \\
\hline & Columbidae & Palomas & Doves & & \\
\hline 18 & Columba livia Gmelin & Paloma doméstica & Rock Dove & $\mathrm{X}$ & \\
\hline 19 & Zenaida auriculata Des Murs & Madrugadora & Eared Dove & $\mathrm{X}$ & $X$ \\
\hline 20 & Zenaida meloda Tschudi & Cuculí & Pacific Dove & $\mathrm{X}$ & $\mathrm{X}$ \\
\hline 21 & Columbina cruziana Prevost & Tortolita peruana & Croaking Ground-Dove & $\mathrm{X}$ & $x$ \\
\hline 22 & Metriopelia ceciliae Lesson & Cascabelita & Bare-faced Ground-Dove & & $\mathrm{X}$ \\
\hline & Psittacidae & Loros y pericos & Parrots & & \\
\hline 23 & Aratinga wagleri Gray & Loro frente roja & Scarlet-fronted Parakeet & & $\mathrm{X}$ \\
\hline 24 & Psilopsiagon aurifrons Lesson & Perico cordillerano & Mountain Parakeet & & $\mathrm{X}$ \\
\hline & Cuculidae & Cuclillos & Cucos & & \\
\hline 25 & Crotophaga sulcirostris Swainson & Guardacaballo & Groove-billed Ani & $x$ & $\mathrm{x}$ \\
\hline & Tytonidae & Lechuza de campanario & Barn owl & & \\
\hline 26 & Tyto alba Scopoli & Lechuza de los campanarios & Barn Owl & $X$ & \\
\hline & Strigidae & Buhos y lechuzas & Owls & & \\
\hline 27 & Glaucidium peruanum Koenig & Paca paca & Peruvian Pygmy-Owl & $\mathrm{X}$ & $X$ \\
\hline 28 & Athene cunicularia Molina & Lechuza de los arenales & Burrowing Owl & $\mathrm{X}$ & $\mathrm{X}$ \\
\hline 29 & Asio flammeus Pontoppidan & Lechuza orejicorta & Short-eared Owl & $\mathrm{X}$ & \\
\hline & Caprimulgidae & Chotacabras & Nightjars & & \\
\hline 30 & Chordeiles acutipennis Hermann & Chotacabra trinador & Lesser Nighthawk & $X$ & $X$ \\
\hline & Apodidae & Vencejos & Swifts & & \\
\hline 31 & Streptoprocne zonaris Shaw & Vencejo cuelliblanco & White-collared Swift & & $X$ \\
\hline 32 & Chaetura pelagica Linnaeus & Vencejo de chimenea & Chimney Swift & $\mathrm{X}$ & $x$ \\
\hline 33 & Aeronautes andecolus Orbigny \& Lafresnaye & Vencejo andino & Andean Swift & $\mathrm{X}$ & $\mathrm{X}$ \\
\hline & Trochilidae & Colibries & Hummingbirds & & \\
\hline 34 & Amazilia amazilia Lesson & Amazilia costeña & Amazilia Hummingbird & $\mathrm{X}$ & $\mathrm{X}$ \\
\hline 35 & Rhodopis vesper Lesson & Colibrí de oasis & Oasis Hummingbird & $\mathrm{X}$ & $\mathrm{X}$ \\
\hline 36 & Thaumastura cora Lesson \& Garnot & Colibrí de Cora & Peruvian Sheartail & $\mathrm{X}$ & $\mathrm{X}$ \\
\hline 37 & Myrtis fanny Lesson & Picaflor de Fanny & Purple-collared Woodstar & $\mathrm{X}$ & $\mathrm{X}$ \\
\hline & Picidae & Carpinteros & Woodpeckers & & \\
\hline 38 & Colaptes atricollis Malherbe & Carpintero peruano & Black-necked Woodpecker & $\mathrm{X}$ & \\
\hline & Furnariidae & Horneros & Ovenbirds & & \\
\hline 39 & Geositta peruviana Lafresnaye & Pampero peruano & Coastal Miner & $\mathrm{X}$ & $X$ \\
\hline 40 & Geositta maritima Orbigny \& Lafresnaye & Pampero gris & Grayish Miner & & $\mathrm{X}$ \\
\hline 41 & Geositta cunicularia Vieillot & Pampero común & Common Miner & $\mathrm{X}$ & $x$ \\
\hline 42 & Leptasthenura striata Philippi \& Landbeck & Tijeral listado & Streaked Tit-Spinetail & & $x$ \\
\hline 43 & Asthenes sp. & Canastero & Spinetail & & $\mathrm{X}$ \\
\hline & Tyrannidae & Mosqueros y tiranos & Flycatchers & & \\
\hline 44 & Camptostoma obsoletum Temminck & Mosquerita silbadora & Southern Beardless-Tyrannulet & $\mathrm{X}$ & $\mathrm{X}$ \\
\hline 45 & Elaenia albiceps Orbigny \& Lafresnaye & Fío fío peruano & White-crested Elaenia & $\mathrm{X}$ & $\mathrm{X}$ \\
\hline 46 & Anairetes reguloides Orbigny \& Lafresnaye & Torito crestipintada & Pied-crested Tit-Tyrant & $\mathrm{X}$ & $\mathrm{X}$ \\
\hline 47 & Anairetes flavirostris Sclater \& Salvin & Torito piquiamarillo & Yellow-billed Tit-Tyrant & & $x$ \\
\hline 48 & Myiophobus fasciatus Statius Muller & Mosquerito pechirrayado & Bran-colored Flycatcher & & $x$ \\
\hline 49 & Euscarthmus meloryphus Wied-Neuwied & Mosqueta copetona & Tawny-crowned Pygmy & $\mathrm{X}$ & \\
\hline 50 & Contopus cinereus Spix & Pibí cenizo & Tropical Pewee & $\mathrm{X}$ & $\mathrm{X}$ \\
\hline 51 & Pyrocephalus rubinus Boddaert & Turtupilín & Vermilion Flycatcher & $\mathrm{X}$ & $\mathrm{X}$ \\
\hline 52 & Ochthoeca leucophrys Orbigny \& Lafresnaye & Pitajo cejiblanca & White-browed Chat-Tyrant & & $\mathrm{X}$ \\
\hline 53 & Muscisaxicola maculirostris Orbigny \& Lafresnaye & Dormilona chica & Spot-billed Ground-Tyrant & & $x$ \\
\hline 54 & Muscisaxicola macloviana Garnot & Dormilona de cabeza oscura & Dark-faced Ground-Tyrant & $\mathrm{X}$ & $x$ \\
\hline 55 & Muscisaxicola albilora Lafresnaye & Dormilona cejiblanca & White-browed Ground-Tyrant & & $\mathrm{X}$ \\
\hline 56 & Muscigralla brevicauda Orbigny \& Lafresnaye & Dormilona cola corta & Short-tailed Field-Tyrant & $x$ & $\mathrm{X}$ \\
\hline 57 & Myiarchus tuberculifer Orbigny \& Lafresnaye & Copetón crestioscura & Dusky-capped Flycatcher & & $\mathrm{X}$ \\
\hline 58 & Lessonia oreas Sclater \& Salvin & Negrito andino & Andean Negrito & $\mathrm{X}$ & \\
\hline 59 & Tyrannus melancholicus Vieillot & Pepite & Tropical Kingbird & $\mathrm{X}$ & $\mathrm{X}$ \\
\hline
\end{tabular}




\begin{tabular}{|c|c|c|c|c|c|}
\hline & Hirundinidae & Golondrinas & Swallows & & \\
\hline 60 & Phaeoprogne tapera Linnaeus & Martín pechipardo & Brown-chested Martin & $X$ & $X$ \\
\hline 61 & Progne murphyi Chapman & Martín peruano & Peruvian Martín & $X$ & $X$ \\
\hline 62 & Pygochelidon cyanoleuca Vieillot & Golondrina Santa Rosita & Blue-and-white Swallow & $X$ & $X$ \\
\hline 63 & Notiochelidon murina Cassin & Golondrina plomiza & Brown-bellied Swallow & & $X$ \\
\hline 64 & Stelgidopteryx ruficollis Vieillot & Golondrina parda & Southern Rough-winged Swallow & $X$ & $X$ \\
\hline 65 & Petrochelidon pyrrhonota Vieillot & Golondrina risquera & Cliff Swallow & $X$ & $X$ \\
\hline 66 & Petrochelidon rufocollaris Peale & Golondrina cuellicastaño & Chestnut-collared Swallow & $X$ & $X$ \\
\hline \multirow[t]{2}{*}{67} & Hirundo rustica Linnaeus & Golondrina tijereta & Barn Swallow & $X$ & $X$ \\
\hline & Motacillidae & Cachirlas & Pipits & & \\
\hline \multirow{2}{*}{68} & Anthus lutescens Pucheran & Chichirre & Yellowish Pipit & & $X$ \\
\hline & Troglodytidae & Cucaracheros & Wrens & & \\
\hline \multirow[t]{2}{*}{69} & Troglodytes aedon Vieillot & Cucarachero & House Wren & $X$ & $X$ \\
\hline & Mimidae & Calandrias & Mockingbirds & & \\
\hline \multirow[t]{2}{*}{70} & Mimus longicaudatus Tschudi & Chisco & Long-tailed Mockingbird & $X$ & $X$ \\
\hline & Passeridae & Gorriones & Old world sparrows & & \\
\hline \multirow[t]{2}{*}{71} & Passer domesticus Linnaeus & Gorrión europeo & House Sparrow & $X$ & $X$ \\
\hline & Fringillidae & Jilgueros & Siskins & & \\
\hline \multirow{2}{*}{72} & Carduelis magellanica Vieillot & Jilguero de cabeza negra & Hooded Siskin & $X$ & $X$ \\
\hline & Parulidae & Reinitas & New world warblers & & \\
\hline \multirow[t]{2}{*}{73} & Geothlypis aequinoctialis Gmelin & Reinita equinoccial & Masked Yellowthroat & & $X$ \\
\hline & Thraupidae & Tangaras & Tanagers & & \\
\hline \multirow{2}{*}{74} & Conirostrum cinereum Orbigny \& Lafresnaye & Mielerito cineréo & Cinereous Conebill & $X$ & $X$ \\
\hline & Emberizidae & Semilleros y espigueros & Buntings \& sparrows & & \\
\hline 75 & Phrygilus alaudinus Kittlitz & Fringilo cola blanca & Band-tailed Sierra-Finch & $X$ & $X$ \\
\hline 76 & Phrygilus $s p$ & Fringilo & Sierra-Finch & $X$ & $X$ \\
\hline 77 & Xenospingus concolor Orbigny \& Lafresnaye & Fringilo apizarrado & Slender-billed Finch & & $X$ \\
\hline 78 & Poospiza hispaniolensis Bonaparte & Dominiquí común & Collared Warbling-Finch & $X$ & $X$ \\
\hline 79 & Volatinia jacarina Linnaeus & Saltapalito & Blue-black Grassquit & $X$ & $X$ \\
\hline 80 & Sporophila simplex Taczanowski & Espiguero simple & Drab Seedeater & $X$ & $X$ \\
\hline 81 & Sporophila peruviana Lesson & Pico grueso & Parrot-billed Seedeater & $X$ & $X$ \\
\hline 82 & Sporophila telasco Lesson & Espiguero corbatón & Chestnut-throated Seedeater & $X$ & $X$ \\
\hline 83 & Catamenia analis Orbigny \& Lafresnaye & Corbatita pico de oro & Band-tailed Seedeater & $X$ & $X$ \\
\hline 84 & Tiaris obscura Orbigny \& Lafresnaye & Semillero pardo & Dull-colored Grassquit & $X$ & \\
\hline 85 & Sicalis olivascens Orbigny \& Lafresnaye & Chirigüe oliváceo & Greenish Yellow-Finch & $X$ & $X$ \\
\hline 86 & Sicalis raimondii Taczanowski & Chirigüe de Raimondi & Raimondi's Yellow-Finch & & $X$ \\
\hline 87 & Atlapetes nationi Sclater & Matorralero ventrirrojizo & Rusty-bellied Brush-Finch & & $X$ \\
\hline \multirow[t]{2}{*}{88} & Zonotrichia capensis Statius Müller & Gorrión americano & Rufous-collared Sparrow & $X$ & $x$ \\
\hline & Cardinalidae & Saltadores & Saltators & & \\
\hline 89 & Saltator striatipectus Lafresnaye & Saltador listado & Streaked Saltator & $X$ & $X$ \\
\hline \multirow[t]{2}{*}{90} & Pheucticus chrysogaster Lesson & Picogrueso ventridorado & Golden-bellied Grosbeak & $X$ & \\
\hline & Icteridae & Vaqueros y oropendolas & New world blackbirds & & \\
\hline 91 & Sturnella bellicosa Filippi & Huanchaco & Peruvian Meadowlark & $X$ & $X$ \\
\hline 92 & Dives warszewiczi Cabanis & Negro & Scrub Blackbird & $X$ & \\
\hline 93 & Molothrus bonariensis Gmelin & Tordo parásito & Shiny Cowbird & $X$ & $\mathrm{X}$ \\
\hline
\end{tabular}

${ }^{1}$ Orden taxonómico de Clements \& Shany (2001). Nombres comunes de Clements \& Shany (2001), Koepcke (1964) y Pulido (1998).

Las especies más abundantes fueron las residentes Zenaida meloda (6,6 ind./ha en promedio por evaluación), Pygochelidon cyanoleuca (5,9 ind./ha) y Mimus longicaudatus (5,1 ind./ha) y la migratoria Hirundo rustica (5,9 ind/ha), juntas alcanzan más del $40 \%$ del total de individuos/ha (Fig. 8).

Zenaida meloda y Mimus longicaudatus son especies típicamente oportunista que se encuentra abundantes en todos los tipos de hábitats y en la mayoría de áreas de cultivo de la costa central del Perú. Por otro lado, Pygochelidon cyanolenca e Hirundo rustica son especies insectívoras que se alimentan en vuelo por encima de las grandes extensiones cultivadas, prestando un importante servicio en la disminución de la abundancia de insectos. Mientras $P$. cyanoleuca presenta una población estable y reproductiva dentro de los fundos, $H$. rustica se presenta en poblaciones muy grandes durante su migración hacia el sur desde Norteamérica (verano austral).

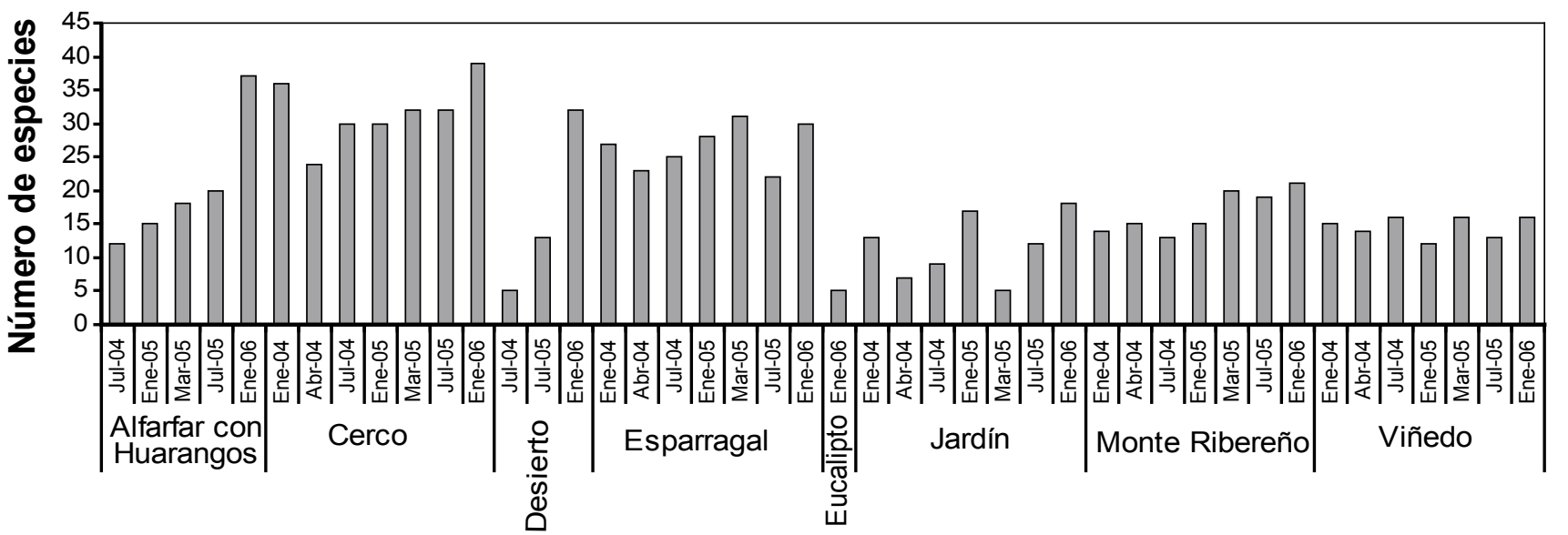

Figura 9. Número de especies para cada periodo de evaluación y en cada tipo de hábitat evaluado. 


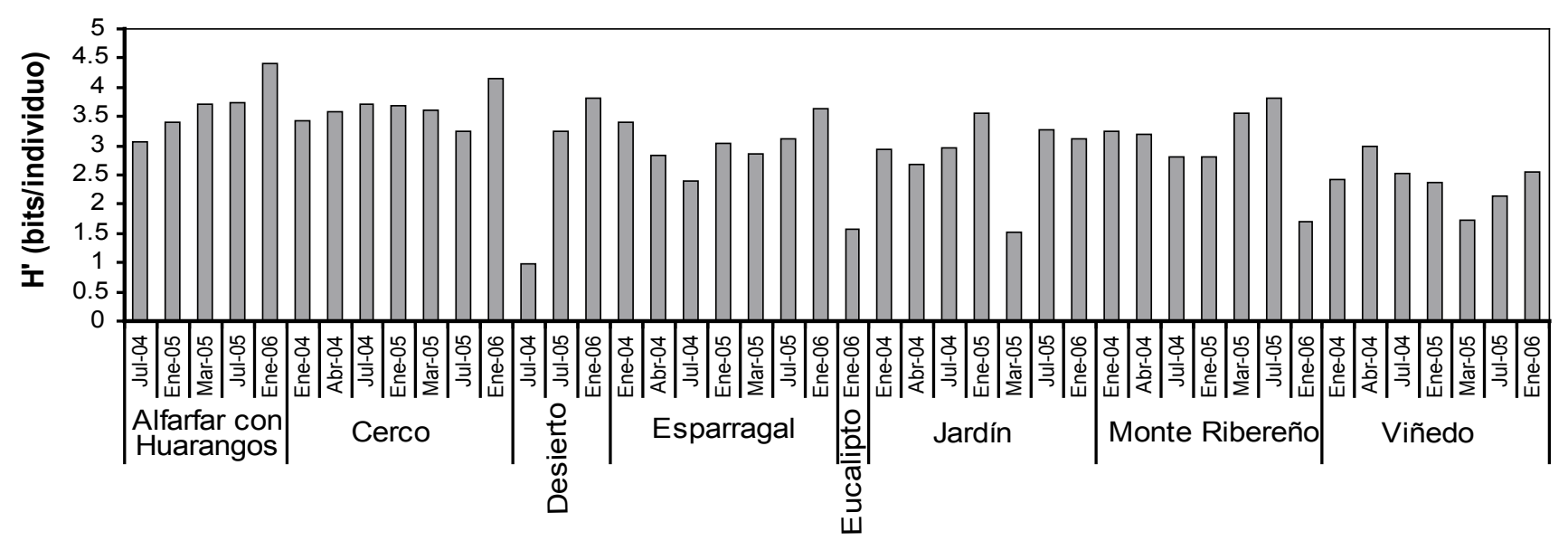

Figura 10. Índice de diversidad de Shannon-Wiener $\left(\mathrm{H}^{\prime}\right)$ para cada periodo de evaluación y en cada tipo de hábitat evalua-

\section{Riqueza y diversidad de especies}

En la gran mayoría de hábitats se encontró un incremento de la riqueza y diversidad en los veranos (Figs. 9 y 10), coincidiendo con la llegada de las especies migratorias en ambos fundos.

Los mayores valores de riqueza y diversidad de especies (índice H' de Shannon-Wiener) ocurrieron en el mes de enero de 2006, en los hábitats de alfalfar con huarangos $\left(\mathrm{H}^{\prime}=4,41\right.$ bits $/$ ind. y 37 especies) y cercos vivos $\left(H^{\prime}=4,14\right.$ bits $/$ ind. y 39 especies) (Figs. 9 y 10).

Estos valores altos de diversidad y riqueza de especies se debe a que el hábitat de alfalfar con huarangos, presenta una importante cobertura arbórea de Prosopis pallida «huarango», además de una mayor variedad de cultivos, de manera que ofrece recursos

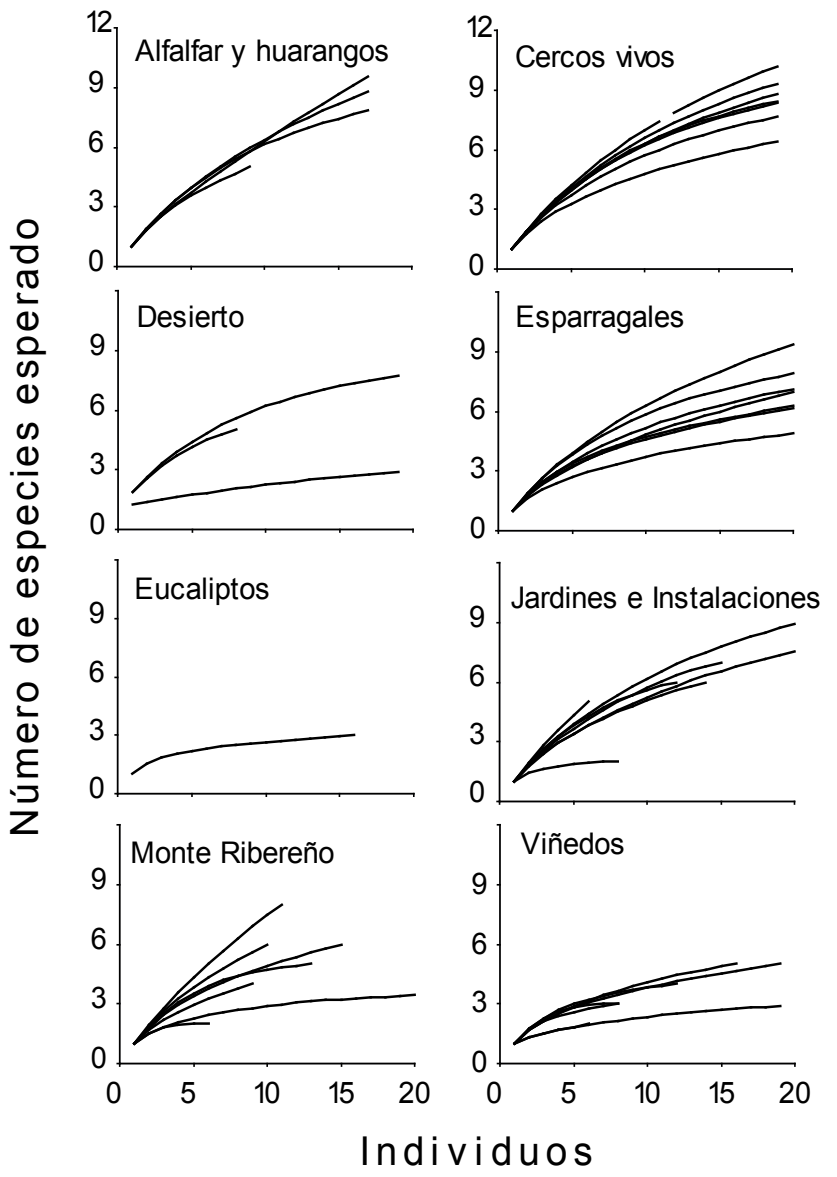

Figura 11. Curvas de rarefacción para cada periodo de evaluación y en cada tipo de hábitat evaluado. únicos en los fundos, atrayendo aves de diferentes especies a pesar de su reducida extensión. En el caso de los cercos vivos, se tratan de hábitats estrechos que se presentan como intermedios entre dos ambientes distintos (dentro y fuera de los fundos), lo que sumado a la presencia de árboles y arbustos atractivos para diferentes especies, incrementa la posibilidad de registro en ellos. Los cercos vivos son en los fundos los lugares con más observaciones de conductas y despliegues reproductivos, habiendo esto contribuido a los altos valores de diversidad de aves en este hábitat durante enero de 2006.

Con la finalidad de evaluar el tamaño de las muestras en los diferentes tipos de hábitats sobre la riqueza de especies, curvas de rarefacción fueron elaboradas para determinar el número de especies esperado para un mismo número de individuos (Fig. 11). En las curvas es notorio observar que los mayores números de especies esperados coinciden los alfalfares con huarangos y cercos vivos, con valores medios para esparragales y jardines e instalaciones, así como valores bajos de número de especies para viñedos y zona de eucaliptos. Las zonas de desierto y monte ribereño muestran una mayor variación en los diferentes meses evaluados.

\section{Especies de interés en conservación}

Tres especies de aves poco frecuentes en los fundos están consideradas amenazadas en la legislación nacional (Decreto Supremo Nº34-2004-AG del 22 de setiembre de 2004), Progne murphy y Xenospingus concolor incluidos en la categoría vulnerable, y Falco peregrinus considerado en la categoría de casi amenazado. El «martín peruano» $P$. murphy es una golondrina del litoral rocoso por lo que su observación en los meses de verano de 2004 es inesperada, pues si bien también se le ha reportado en áreas cultivadas y zonas urbanas, los dos fundos se encuentran bastante alejados del mar. Es de especial interés la presencia del «fringilo apizarrado» $X$. concolor cuya población residente habita los parches de árboles y arbustos dispersos en la zona del desierto de la Catalina, manteniéndose en la reserva de desierto de AGROKASA, principalmente en los inviernos. Hemos observado otra población importante de este fringilo en los oasis de Alto Bernal y de Morón en el valle del Pisco, donde se establece en la vegetación dominada por el arbusto Pluchea chingoyo (Kunth) DC. Estas observaciones indican que esta especie ocupa una mayor variedad de ambientes que lo anteriormente reportado (Gonzáles \& Málaga, 1997b). La mayoría de los registros del «halcón peregrino» $F$. peregrinus corresponde a una población reproductiva habiéndose observado varias parejas en el fundo. 


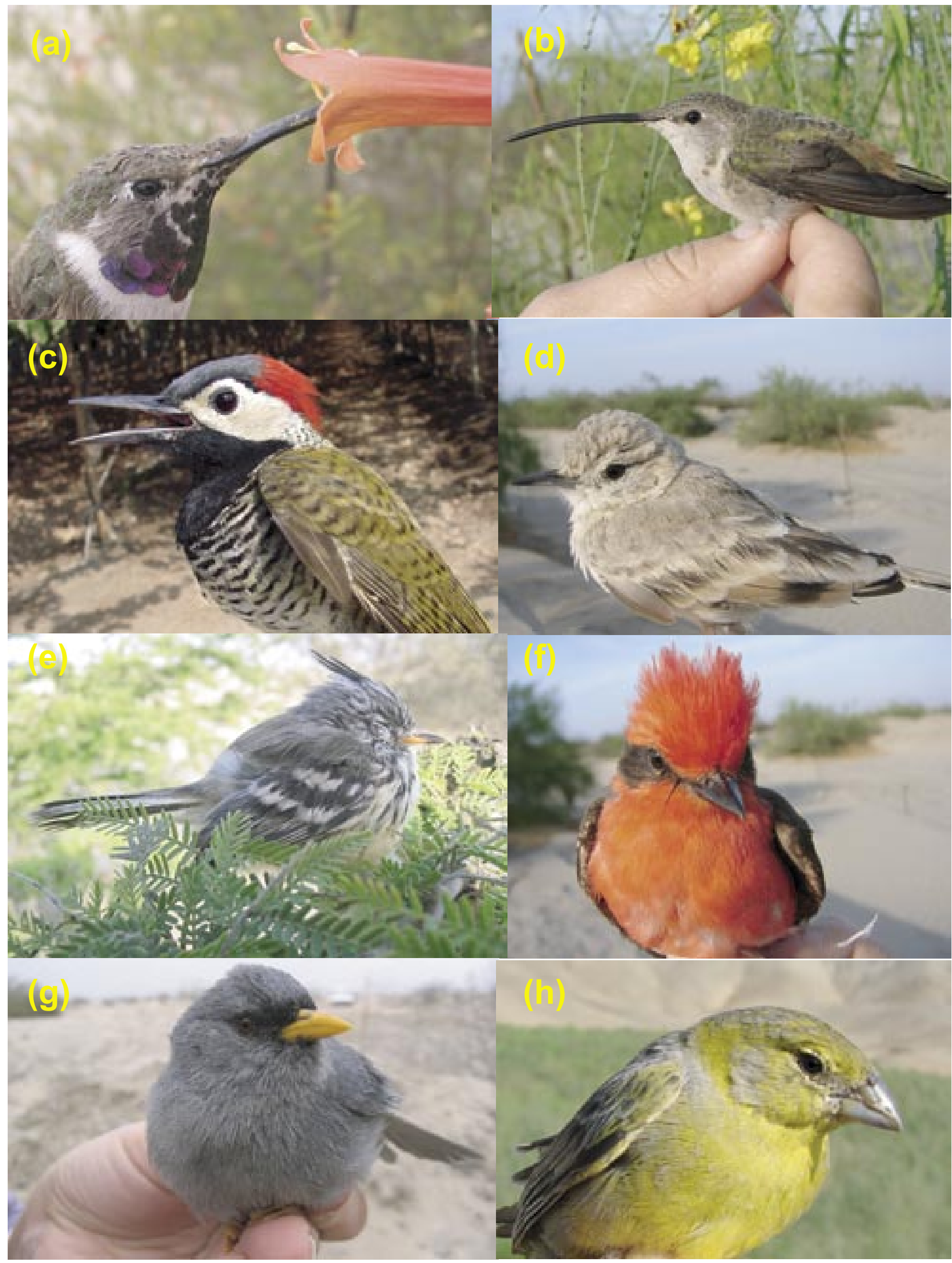

Figura 12. Algunas especies de aves representativas de los fundos de AGROKASA en Ica. (a) Rhodopis vesper «colibrí de oasis» (ejemplar macho alimentándose de Tecoma guarume); (b) R. vesper hembra; (c) Colaptes atricollis "carpintero peruano»; (d) Geositta peruviana "pampero peruano»; (e) Anairetes flavirostris «torito piquiamarillo», (f) Pyrocephalus rubinus «turtupilín», (g) Xenospingus concolor «fringilo apizarrado»; (h) Sicalis raimondii «Chirigüe de Raimondi». 
Cuatro especies de aves, el «carpintero peruano» Colaptes atricollis, el «pampero peruano» Geositta peruviana, el «chirigüe de Raimondi» Sicalis raimondii y el «matorralero ventrirrojizo» Atlapetes nationi, son endémicas del Perú. Entre estas especies destacan, G. peruviana con una población abundante y residente dentro de los fundos con tendencia al incremento de sus poblaciones, y $C$. atricollis registrado principalmente en la vegetación ribereña que rodea los grandes canales de regadío que cruzan el fundo Santa Rita y en los cercos vivos aledaños al río Ica.

El «martín peruano» Progne murphyi y el «fringilo apizarrado» Xenospingus concolor adicionalmente, están incluidas en la lista roja de IUCN (2006), en las categorías de Vulnerable y Casi amenazada, respectivamente.

Las Áreas de Endemismo de Aves (EBAs) constituyen una de las prioridades mundiales de conservación de la biodiversidad porque contienen un importante número de especies de aves y grupos de flora y fauna valiosas, que se encuentran exclusivamente en una región menor a $50000 \mathrm{~km}^{2}$ (Stattersfield et al., 1998). En los fundos de AGROKASA, cuatro especies de aves se encuentran incluidas en las EBAs: el «pampero peruano» Geositta peruviana y el «espiguero simple» Sporophila simplex de las EBAs 045 y 052 (Tumbesian region y Peru-Chile Pacific slope, respectivamente); el «fringilo apizarrado» Xenospingus concolor es indicador de la EBA 052; y el «matorralero ventrirrojizo» Atlapetes nationi indicador de la EBA 051 (Peruvian High Andes). Las dos primeras especies son las únicas con poblaciones residentes y abundantes dentro de los fundos.

Otro acercamiento a la detección de especies de aves importantes en conservación es la identificación de algunas que son exclusivas de regiones extensas y homogéneas, con formas de vida características y especies vegetales propias llamadas Biomas (o Regiones zoogeográficas, sensu Stotz et al., 1996). En la avifauna de los fundos se han encontrado siete especies indicadoras de biomas. Una de ellas es el «fringilo apizarrado» Xenospingus concolor indicadora del Bioma Pacífico subtropical (STP: Subtropical Pacific); la mayoría sin embargo, son indicadoras del bioma Andes Centrales (CAN) mostrando la fuerte influencia de la avifauna de las vertientes occidentales andinas sobre estos fundos ubicados cerca de las primeras estribaciones andinas. Estas especies son, la «cascabelita» Metriopelia ceciliae, el «tijeral listado» Leptasthenura striata, el «negrito andino» Lessonia oreas, el «chirigüe de Raimondi» Sicalis raimondii y el «matorralero ventrirrojizo» Atlapetes nationi. La «dormilona cejiblanca» Muscisaxicola albilora, indicadora del bioma Andes del Sur (SAN) ubicado fuera del Perú, ha sido registrada en La Catalina durante su migración hacia el norte del subcontinente durante el otoño e invierno de 2003.

Las especies de los órdenes Falconiformes, Psittaciformes y Strigiformes, así como de la familia Trochilidae están consideradas en el Apéndice II de la Convención sobre el Comercio Internacional de Especies Amenazadas de Fauna y Flora Silvestres (CITES), sólo el «halcón peregrino» Falco peregrinus se encuentra en el Apéndice I.

Doce especies de aves de las observadas suelen presentar poblaciones migratorias: el «aguilucho ala ancha» Buteo platypterus, el «halcón peregrino» Falco peregrinus, el «chorlo gritón» Charadrius vociferus, el «chorlo nevado» Charadrius alexandrinus, el «vencejo de chimenea «Chaetura pelagica, la "golondrina parda» Stelgidopteryx ruficollis, la «golondrina risquera» Petrochelidon pyrrbonota, la «go- londrina tijereta» Hirundo rustica, el «chorlo de campo» Oreopholus ruficollis, la «dormilona de cabeza oscura» Muscisaxicola macloviana, la «dormilona cabeciblanca» Muscisaxicola albilora y el «martín pechipardo» Phaeoprogne tapera. Las ocho primeras especies con poblaciones reproductivas en Norteamérica y las últimas cuatro con poblaciones reproductivas en el sur de Sudamérica. Sin embargo, ya que el Perú es un país tropical donde no ocurren cambios estacionales extremos del clima, cuatro especies presentan poblaciones reproductivas en la costa peruana (Falco peregrinus, Charadrius vociferus, Charadrius alexandrinus y Oreopholus ruficollis).

Cinco especies migratorias están incluidas en el Apéndice II de la Convención sobre la Conservación de las Especies Migratorias (CMS, 1979), el «aguilucho ala ancha», el «halcón peregrino», el «chorlo gritón», el «chorlo nevado» y el «chorlo de campo». Sin embargo, cuatro de las especies presentan poblaciones residentes en la región, lo que las excluye de la Convención, quedando únicamente en esta categoría el «aguilucho ala ancha» Buteo platypterus. Cuatro individuos de este aguilucho fueron observados sobrevolando ambos fundos en agosto de 2003.

Tres especies de golondrinas migratorias, visitantes de verano, se muestran particularmente importantes, la «golondrina tijereta» Hirundo rustica, el «martín pechipardo» Phaeoprogne tapera y la «golondrina risquera» Petrochelidon pyrrhonota, cuyas poblaciones en su conjunto forman conglomerados de casi dos mil individuos (Enero de 2006).

Las especies «congregatorias» son aves acuáticas, marinas o terrestres que reúnen una fracción importante de su población global en ciertos lugares como colonias de anidación, sitios de parada o descanso, terrenos de hibernación o lugares de concentración en su recorrido migratorio (BirdLife Internacional \& Conservation Internacional, 2005). La concentración de al menos $1 \%$ de la población global de las especies congregatorias es considerada como uno de los criterios para el establecimiento de los sitios Ramsar (humedales de importancia internacional protegidos bajo la Convención Ramsar) y para la determinación de las IBAs (Áreas Importantes para la Conservación de las Aves, según BirdLife International) en todo el mundo. En los fundos de AGROKASA se observaron ocho especies congregatorias. Seis de ellas son acuáticas pero sus poblaciones en los fundos son menores que los valores reconocidos como de importancia internacional (1\% de la población global). Estas especies son la «garza blanca grande» Ardea alba, la «garza blanca chica» Egretta thula, la «gallineta común» Pardirallus sanguinolentus, la «polla de agua» Gallinula chloropus, el «chorlo nevado» Charadrius alexandrinus y el «chorlo gritón» Charadrius vociferus.

Dos poblaciones reproductivas de especies congregatorias terrestres fueron registradas en los fundos. La primera es la del «huerequeque» Burbinus superciliaris, con abundancia significativamente mayores a 100 individuos, cifra que representa el 1\% de su población global. El «huerequeque» ha sido registrado con poblaciones reproductivas en los dos fundos de AGROKASA, pero su mayor población ha sido estimada para La Catalina, donde es más frecuentemente oída y observada. La segunda es la del «chorlo de campo» Oreopholus ruficollis, con una población menor al 1\% de su población global (200 individuos) en la zona desértica sin cultivar de La Catalina, en donde existe un único grupo familiar de no más de 15 individuos. 
El agroecosistema evaluado presenta varias y valiosas especies que pueden ser de interés en el programa Áreas Importantes para la Conservación de las Aves (IBAs) de BirdLife Internacional (Franke et al., 2005), que identifica estas áreas por la presencia de especies que cumplen ciertos criterios (Fig. 12). Dos especies presentes en el fundo cumplen con el criterio A1 (especies globalmente amenazadas) Xenospingus concolor y Progne murphyi, cuatro con el criterio A2 (especies de distribución restringida o de EBAs) Geositta peruviana, Sporophila simplex, Xenospingus concolor y Atlapetes nationi. Además, se presentan siete especies indicadoras de biomas en el criterio A3 y ocho especies congregatorias para el criterio A4. De todas estas especies sólo tres son significativamente importantes Geositta peruviana, Sporophila simplex y Burbinus superciliaris que presenta poblaciones abundantes y en crecimiento a las cuales se esta realizando un seguimiento particular en los fundos.

En el mundo la mayoría de las IBAs coinciden con unidades de conservación bajo protección internacional, nacional o son reservas indígenas o comunales. Sin embargo, existen algunas pocas propuestas que incluyen áreas cultivadas, como las existentes en los arrozales de Cuba (Mugica, 2005). Si bien los fundos de AGROKASA no alcanzan todos los requisitos para la calificación como una IBA, se acercan mucho a cumplirlos, ya que se pueden encontrar especies en todos los criterios para la definición de éstas áreas.

Las IBAs para el Perú han sido definidas en un proceso participativo culminado en el 2004 (Franke et al., 2005) y no existe un área con características ornitológicas similares a las de los fundos. En consecuencia es importante señalar que políticas de respeto de la diversidad biológica como las de AGROKASA, revierten en la conservación de las escasamente protegidas aves del desierto costero.

\section{Discusión}

Los fundos de AGROKASA están ubicados en el desierto costero, una región naturalmente de baja diversidad biológica. Sin embargo, el número total acumulado de especies de aves es muy alto en comparación con los estudios realizados en otros ambientes costeros del Perú, lo que refleja tanto la intensidad del estudio como la especial riqueza de este ambiente. Por ejemplo, en la Reserva Nacional Lomas de Lachay (Lima) se reportan 55 especies (Wust, 1987), en el valle del río Majes (Arequipa) 53 (Gonzáles y Málaga, 1997a), en las lomas de Atiquita (Arequipa) 59 (Zeballos et al., 2000), en la cuenca baja del río Piura (Piura) 72 (More y Cadenillas, 2004), en el humedal de El Paraíso (Lima) 81 (Cruz et al. en prensa) y en los humedales de Villa (Lima), los únicos ambientes evaluados durante más de un siglo, 186 especies (Pulido, 2003).

Esta riqueza de especies de aves se debe a que en los fundos de AGROKASA se han implementado varias medidas, identificadas en otros agroecosistemas (Tilman, 1999) como importantes para la conservación de la biodiversidad silvestre, incluyendo la preservación de áreas con vegetación silvestre aledaña, uso cuidadoso de agroquímicos, control biológico de plagas e incremento de heterogeneidad en el paisaje, utilizando especies de plantas silvestres como cercos vivos. Adicionalmente, el riego por goteo permitió conservar grande extensiones de suelo desértico arenoso favoreciendo la presencia de aves propias del desierto, como pamperos, huerequeques y lechuzas de los arenales.
Las plantas silvestres son de especial importancia porque conforman diversos estratos (hierbas, arbustos y árboles) que se constituyen en el hábitat y fuente de recursos alimenticios para las aves (néctar, granos, semillas y frutos). En los fundos evaluados los cercos están principalmente conformados por «aromo» Acacia cf. horrida, una especie introducida de África (Pérez et al., 2002), sin embargo esta planta presenta una estructura similar a las especies nativas de Acacia. A esta característica se le suma la presencia de algunos árboles de «huarango» Prosopis pallida y de otras especies, que contribuyen de manera significativa al incremento de la complejidad estructural.

La complejidad en la estructura vegetal resultó ser un elemento particularmente importante en estos fundos, observándose que la mayor variedad de especies de aves se encuentran en los hábitats de alfalfar con huarangos y en los cercos vivos, los ambientes de mayor diversidad estructural. Durante el periodo de estudio además del mantenimiento de las plantas de los cercos y erradicación de eucaliptos de sectores del mismo, ocurrió un progresivo enriquecimiento con especies vegetales nativas lo que contribuyó a un incremento significativo de las especies de aves estrechamente ligadas a la vegetación silvestre.

El relativamente alto número de especies de aves rapaces, como águilas y lechuzas residentes en los fundos es un buen indicador del adecuado manejo de insectos plaga realizado principalmente en base al control biológico sin perjuicio de las poblaciones de vertebrados pequeños, como ha sido comprobado en otros países (Belgrafe et al., 2005). El nivel trófico alto que ocupan las rapaces como depredadoras las hace indicadoras de la abundancia de presas como aves pequeñas, roedores, lagartijas o pequeñas culebras, así como vulnerables a la bioacumulación de insecticidas, como ha sido probado en diferentes ecosistemas (Wiemeyer et al., 1984; Helander et al., 2002; Hernández et al., 1988; Scharenberg, 2004). De modo tal, que la alta diversidad de especies carnívoras de aves en estos fundos son resultado del uso responsable de agroquímicos.

El esfuerzo por la conservación de la biodiversidad realizado en el Perú se ha concentrado en el establecimiento de Áreas Naturales Protegidas. Sin embargo, necesitamos explorar otras formas de conservación in situ. El manejo sustentable de agroecosistemas podría ser una opción válida para la conservación de especies, pero que aún no ha sido evaluada exhaustivamente (Belfrage et al., 2005; Chiarello, 2000; Tilman, 1999; Mugica, 2005; Vandermeer \& Perfecto, 1997), y que en muchas regiones del Perú podría tener un impacto positivo en la protección de la biota silvestre.

La conservación de la avifauna en los agroecosistemas proporciona varios beneficios para los agricultores. Por un lado un gran porcentaje de las especies de aves presentes son de hábitos alimentarios insectívoros convirtiéndose en controladores naturales de plagas, como ha sido documentado para cultivos de arroz, sorgo y quinua (Poleo \& Fuentes, 2005; Robles et al., 2003). Adicionalmente, muchas especies son polinizadoras de especies de interés económico y otras disminuyen malezas consumiendo sus semillas (Beal, 1899; Myers, 1996; Robles et al., 2003; Cuevas, 2001).

En el presente estudio mostramos que los agroecosistemas de los desiertos costeros poseen un importante potencial de conservación de la avifauna local; este potencial estaría relacionado 
al compromiso de una política empresarial de manejo ordenado, tecnificado y ambientalmente respetuoso. Estas normas de respeto al ambiente deben ser asumidas no sólo como parte de una política de responsabilidad social, sino que las nuevas tendencias del mercado internacional al cual esta dirigido las exportaciones agrícolas exigen que la producción sea ambientalmente saludable (FAO, 2004; Stefanelo, 2002; Scialabba \& Hattam, 2003), por lo que preservar la biodiversidad resulta un buen negocio.

\section{Agradecimientos}

Expresamos nuestro reconocimiento a la Sociedad Agrícola Drokasa (AGROKASA) por las facilidades y financiamiento otorgado para el presente estudio. En particular al Presidente del Directorio Ing. J. Chlimper, al Gerente General Ing. C. Arrese y al Director de la Corporación Drokasa Dr. J. Callaux. Agradecemos al Ing. J. Briceño por las facilidades logísticas brindadas. A María del Carmen Samamé que nos acompaño en la primera evaluación de campo, nuestro afecto y aprecio. También nuestro agradecimiento a los trabajadores de los fundos Santa Rita y La Catalina por sus muestras de aprecio en cada una de nuestras visitas.

\section{Literatura citada}

Belfrage, K.; J. Björklund \& L. Salomonsson. 2005. The Effects of Farm Size and Organic Farming on Diversity of Birds, Pollinators, and Plants in a Swedish Landscape. AMBIO: A Journal of the Human Environment 34(8):582-588.

Bibby, C.J.; N.D. Burgers \& D.A. Hill. (1993). Bird Census Techniques. Academic Press, Cambridge.

Bibby, C.J. \& T.D. Charlton. 1991. Observation on the San Miguel Bullfinch. Acoreana 7: 297-304

Bibby, C.J.; B.N. Phillips, A.J. Seddon. 1985. Birds of restoked conifer plantations in Wales. Journal of Applied Ecology. 22: 619-633

BirdLife Internacional \& Conservación Internacional. (2005). Áreas Importantes para la Conservación de las Aves en los Andes Tropicales: sitios prioritarios para la conservación de la biodiversidad. Quito, Ecuador. Serie de Conservación de BirdLife No. 14.

Buckland, S.T. 1987. On the variable circular plot method of estimating density. Biometrika. 43: 363-384.

Chiarello, A.G. 2000. Conservation value of a native forest fragment in a region of extensive agriculture. Rev. Brasil. Biol. 60(2):237-247.

CITES. 2006. (en línea) Convención sobre el Comercio Internacional de Especies Amenazadas de Fauna y Flora Silvestres. Apéndices I,II y III. <htp://www.cites.org/esp/app/appendices.shtml>. Acceso el 1/07/2006.

Clements, J. \& Shany, N. (2001). Birds of Peru. Ibis Publishing Company. California.

CMS. 1979. (en línea) Convención sobre la Conservación de las Especies Migratorias de Animales Silvestres. $<$ http://www. $\mathrm{cms}$.int/documents/convtxt/cms_convtxt_sp.htm>. Acceso $31 / 01 / 2007$.

Cruz, Z.; F. Angulo, H. Burger y R. Borgesa. (en prensa). Evaluación de Aves en la Laguna El Paraíso, Lima, Perú. Revista Peruana de Biología.

Cuevas, M.A. 2001. Aves migratorias: constante amenaza para el cultivo del arroz. Arroz (Colombia): 50(432):16-19.

Curran, L.M., S.N. Trigg, A.K. McDonald, D. Astiani, Y.M. Hardiono, P. Siregar, I. Caniago \& E. Kasischke. 2004. Lowland forest loss in protected areas of Indonesian Borneo. Science 303:1000-1003.
Daily, G.C., P.R. Ehrlich \& G.A. Sanchez-Azofeifa. 2001. Country biogeography: use of human-dominated habitats by the avifauna of southern Costa Rica. Ecological Applications $11: 1-13$

Defries, R.S., J.A. Foley \& G.P. Asner. 2004. Land-use choices: balancing human needs and ecosystem function. Frontiers in Ecology and the Environment 2:249-257.

Estrada, A., A. Anzures \& R. Coates-Estrada. 1999. Tropical rain forest fragmentation, howler monkeys (Aloutta palliata), and dung beetles at Los Tuxtlas, Mexico. American Journal of Primatology 48:253-262.

Estrada, A., R. Coates-Estrada, A.A. Dadda \& P. Cammarano. 1998. Dung and carrion beetles in tropical rain forest fragments and agricultural habitats at Los Tuxtlas, Mexico. Journal of Tropical Ecology 14:577-593.

Food and Agriculture Organization of the United Nations (FAO). 2004. The State of Agricultural Commodity Markets. Editorial Production and Design Group Publishing Management Service, FAO, Roma.

Franke, I.; J. Mattos, L. Salinas, C. Mendoza \& S. Zambrano. 2005. Áreas Importantes para la Conservación de las Aves en Perú. Pp. 471-510 en BirdLife Internacional y Conservación Internacional. Áreas Importantes para la Conservación de las Aves en los Andes Tropicales: sitios prioritarios para la conservación de la biodiversidad. Quito, Ecuador. Serie de Conservación de BirdLife 14.

Fujisaka, S., S. Escobar \& E. Veneklaas. 1998. Plant community diversity relative to human land uses in an Amazon forest colony. Biodiversity and Conservation 7:41-57.

Gonzáles, O. \& E. Málaga. 1997a. Distribución de aves del valle de Majes, Arequipa, Perú. Ornitología Neotropical 8:57-69.

Gonzáles, O. \& E. Málaga. 1997b. Estudios preliminaries de la ecología de Xenospingus concolor en el Perú. Actas del III Encuentro Bolivariano para la Conservación de las Aves. Bolivia. Pp. 47-48.

Harvey, C.A., C. Villanueva, J. Villacis, M. Chacon, M. Munoz, M. Lopez, M. Ibrahim, R. Gomez, R. Taylor, J. Martinez, A. Navas, J. Saenz, D. Sanchez, A. Medina, S. Vilchez, B. Hernandez, A. Perez, E. Ruiz, F. Lopez, I. Lang \& F.L. Sinclair. 2005. Contribution of live fences to the ecological integrity of agricultural landscapes. Agriculture, Ecosystems and Environment 111:200-230.

Helander, B., A. Olsson, A. Bignert, L. Asplund, K. Litzén. 2002. The Role of DDE, PCB, Coplanar PCB and Eggshell Parameters for Reproduction in the White-tailed Sea Eagle (Haliaeetus albicilla) in Sweden. AMBIO: A Journal of the Human Environment 31(5):386-403.

Hernández, L.M.; Ma.J. González, Ma.C. Rico, M.A. Fernández $\&$ A. Aranda. 1988. Organochlorine and heavy metal residues in Falconiforme and Ciconiforme eggs (Spain). Bulletin of Environmental Contamination and Toxicology 40(1):86-93.

Heywood, V.H. \& R.T. Watson (eds.) (1995). Global biodiversity assessment. Cambridge University Press, Cambridge, UK.

INEI. 2007. (en línea). Información Económica. <http://www.inei. gov.pe $>$. Acceso 31/01/2007.

IUCN. 2006. (en línea) 2006. IUCN Red List of Threatened Species. The World Conservation Union. <http://www.iucnredlist. org/>. Acceso 31/01/2007.

Judd, S.D. 1898. Birds as Weed Destroyers. U.S. Department of Agriculture, Washington. Pp. 221-232.

Koepcke, M. (1964). Las Aves del Departamento de Lima. Edit. Morson. Lima.

Koskimies, P. \& R.A. Vaisanen (eds.) 1991. Monitoring Bird populations. Finniish Museum of Natural History, Helsinki. 
Magurran, A.E. (1991). Ecological Diversity and its Measurement. Chapman \& Hall. New York.

Málaga, E. \& R. Rayán. 2003. Aves que afectan el cultivo de la uva en la irrigación Majes, Arequipa, Perú. Dilloniana 3(1):89.

Mayfield, M.M. \& G.C. Daily. 2005. Countryside biogeography of neotropical herbaceous and shrubby plants. Ecological Applications 15:423-439.

Ministerio de Agricultura.Decreto Supremo No. 034-2004-AG. 2004. EL PERUANO Pp. 276853-276855.

Ministerio de Agricultura. 2007. (en línea). Noticias agrarias. $<\mathrm{http} / /$ www.minag.gob.pe/noticias_agraria.shtml? $x=67750>$. Acceso 31/01/2007.

More, A. y R. Cadenillas. 2004. Aves de la cuenca baja del río Piura, inventario preliminar. Dilloniana 4(1):118-121.

Mugica, L. 2005. Las arroceras y humedales: dos propuestas de IBAs en Cuba. BirdLife Caribbean 3:9-10.

Myers, N. 1996. The Biodiversity Crisis and the Future of Evolution. The Environmentalist 16: 1-11.

Myers, N., R.A. Mittermeier, C.G. Mittermeier, G.A.B. Da Fonseca \& J. Kent. 2000. Biodiversity hotspots for conservation priorities. Nature, 403, 853-858.

O’Brien, T.M. \& A. Díaz. 2004. Mejorando la competitividad y el acceso a los mercados de exportaciones agrícolas por medio del desarrollo y aplicación de normas de inocuidad y calidad: El ejemplo del espárrago Peruano. Reporte del Programa de Sanidad Agropecuaria e Inocuidad de Alimentos del Instituto Interamericano de Cooperación para la Agricultura (IICA), Costa Rica. Pp. 1-27.

Ormerod, S.J. \& A.R. Watkinson. 2000. Birds and agriculture - Editor's introduction. Journal of Applied Ecology 37:699-705

Pérez, R.B.; C. Reynel \& M. Manta. 2002. Dendrología y propagación vegetativa de Acacia horrida («Huaranguillo») Mediante estacas inducidas en tres sustancias enraizantes, usando tres sustratos. Ecología Aplicada 1(1):9-12.

Pilares, P. \& M. Arenas. 2004. Magnitud del daño que ocasionan las aves en los cultivos de arroz circundantes a la zona de amortiguamiento (Chical bajo) del Santuario Nacional Lagunas de Mejía. Dilloniana 4(1):90-91.
Poleo, C. \& L. Fuentes. 2005. (en línea) Aves plagas de los cultivos de arroz y sorgo en Venezuela. Revista Digital del Centro Nacional de Investigaciones Agropecuarias de Venezuela 9. $<\mathrm{http} /$ www.ceniap.gov.ve/ceniaphoy/articulos/n9/arti/ poleo_c/arti/poleo_c.htm $>$. Acceso 17/10/2006.

Pulido, V. (1998). Vocabulario de los nombres comunes de la fauna silvestre del Perú. Lima.

Pulido, V. 2003. Influencia de la pérdida de hábitats en la conservación de las aves de los Pantanos de Villa. Tesis para optar el grado Académico de Doctor en Ciencias Biológicas. UNMSM.

Reyes, R. 1985. Pájaros que se alimentan del grano de sorgo en maduración, San Andrés, La Libertad, El Salvador. CENTA, Boletín Informativo 25:7-8.

Reynolds, R.T.; J.M. Scott \& R.A. Nussbaum. 1980. A variable circular plot method for estimating birds numbers. Condor 82: 309-313.

Robles, J.S-E.; C. Rasmussen, V. Otazu, Mandujano. 2003. Plagas de aves en quinua (Chenopodium quinoa Willd.) y medidas de control en el Perú central. Rev. per. Ent. 43: 147-150.

Scharenberg, W. 2004. Reduction of Organochlorine Residues in Goshawk Eggs (Accipiter gentilis) from Northern Germany (1971-2002) and Increasing Eggshell Index. AMBIO: A Journal of the Human Environment: 33(8):495-498.

Schroth, G., G.A.B. Da Fonseca, C.A. Harvey, C. Gascon, H.L. Lasconcelos \& A.-M.N. Izac. 2004. Agroforestry and biodiversity conservation in tropical landscapes. Island Press, Washington, D.C.

Scialabba, N.E. \& C. Hattam. (Eds.) 2003. Agricultura Orgánica, Ambiente y Seguridad Alimentaria. 280 pp, 16 figuras, 21 cuadros, Colección FAO: Ambiente y Recursos Naturales $\mathrm{N}^{\circ} 4$, Roma.

Settele, J., K. Martin \& T. Achilles. 1993. Philippine rice terraces - Investigations into the fauna of a traditional agroecosystem and the effects of agriculture change. In: W. Barthlott, C.M. Naumann, K. Schmidt-Loske \& K.-L. Schuchmann (eds.) Animal-Plant Interactions in Tropical Environments. Zoologische Forschungsinstitut und Museum Alexander Koenig, Bonn. Pp. 219-227.

Stefanelo, E.L. 2002. Agronegócio brasileiro: propostas e tendências. FAE Business 3:10-13. 


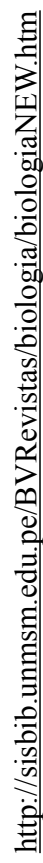

